\title{
La problématique du stockage des déchets ménagers et assimilés face au changement d'échelle de planification en Région Île-de-France
}

The question of the landfilling of non-dangerous waste faced with the change of scale planning in the Ile-de-France region

\section{Rym Mtibaa, Jacques Mery et André Torre}

\section{OpenEdition \\ Journals}

\section{Édition électronique}

URL : https://journals.openedition.org/developpementdurable/9451

DOI : 10.4000/developpementdurable.9451

ISSN : 1772-9971

\section{Éditeur}

Association DD\&T

\section{Référence électronique}

Rym Mtibaa, Jacques Mery et André Torre, «La problématique du stockage des déchets ménagers et assimilés face au changement d'échelle de planification en Région Île-de-France », Développement durable et territoires [En ligne], Vol. 3, n 3 | Décembre 2012, mis en ligne le 25 juillet 2014, consulté le 21 septembre 2021. URL : http://journals.openedition.org/developpementdurable/9451; DOI : https:// doi.org/10.4000/developpementdurable.9451

Ce document a été généré automatiquement le 21 septembre 2021.

\section{(c) $(1) \otimes$}

Développement Durable et Territoires est mis à disposition selon les termes de la licence Creative Commons Attribution - Pas d'Utilisation Commerciale 4.0 International. 


\section{La problématique du stockage des déchets ménagers et assimilés face au changement d'échelle de planification en Région Île-de- France}

The question of the landfilling of non-dangerous waste faced with the change of scale planning in the Ile-de-France region

Rym Mtibaa, Jacques Mery et André Torre

Ce travail a été financé par le programme R2DS de la région Ile de France

1 Fédéralisme, principe de subsidiarité, théorème de la localité, proximité, dilemme efficacité/équité, toutes ces expressions traduisent chacune à leur manière la question générale des échelles territoriales de gestion pertinentes, voire optimales pour les politiques publiques. Nous présentons ici le cas particulier de la gestion des déchets sur le territoire francilien, passée à la fin des années 2000 de l'échelle départementale à l'échelle régionale, avec un focus sur des installations problématiques du fait qu'elles consomment beaucoup d'espace et qu'elles sont source de certaines nuisances, les Installations de Stockage de Déchets Non Dangereux (ISDND). Alors que différents rapports nationaux tirent le signal d'alarme sur le risque de pénurie d'exutoires pour le traitement des déchets résiduels (SENAT, 2010; MEEDDM, 2009; ADEME, 2007), le cas de l'Île-de-France semble un peu moins préoccupant depuis que les deux sites les plus importants de la région ${ }^{1}$ ont vu leurs autorisations d'exploitation prolongées pour 20 ans, en 2006 et 2007. Ces décisions reporteraient donc la problématique d'une éventuelle situation de crise dans le long terme et exempteraient de ce fait les autorités en charge de la planification de la gestion des déchets de se prononcer sur la question du choix de taille et de localisation des ISDND. 
2 Mais est-ce si sûr? En effet, le temps moyen qui s'écoule entre l'initiation d'un projet de nouvelle installation et son autorisation par la préfecture est de l'ordre d'une décennie, soit autant que l'horizon temporel de la planification territoriale de la gestion des déchets, et se prolonge à mesure que les mouvements d'opposition locale s'organisent et se déclenchent de plus en plus tôt, comme face à la plupart des projets d'infrastructures (Torre, 2010). La capitalisation des expériences par les acteurs du conflit, aidés par les nouvelles technologies de l'information, permet aux associations de défense de l'environnement et du cadre de vie de s'organiser efficacement. Le problème ne serait donc pas d'être envahi de déchets mais de ne pas pouvoir trouver des sites $\mathrm{d}^{\prime}$ enfouissement à cause du caractère localement indésirable de ces derniers ${ }^{2}$ (Lomborg, 2004) et des exigences foncières spécifiques aux ISDND (Méry, Mtibaa et Torre, 2009).

Dans ces conditions, pour une des régions les plus peuplées d'Europe, la possibilité que ces deux sites d'enfouissement ferment après plus de 54 ans d'exploitation est donc un problème à anticiper. En dépit des efforts réalisés au niveau de tous les leviers de la gestion des déchets (prévention, tri, recyclage, valorisation, etc.), il restera toujours un déchet "ultime" pour des raisons thermodynamiques (Baumgärtner et de Swans Arons, 2003). Un constat que reprend le Sénat dans son rapport d'information sur le traitement des déchets: «quelles que soient les performances atteintes grâce à la valorisation matière ou énergétiques, les modes de prétraitement ou de traitement des ordures ménagères génèrent des déchets ultimes pour lesquels le stockage demeure la seule solution techniquement possible » (SENAT, 2010). Or, les contacts avec les responsables régionaux confirment qu'il sera à l'avenir impossible d'autoriser des ISDND de la taille des actuelles infrastructures de Claye-Souilly (77) ou du Plessis-Gassot (95). Dans l'éventualité où ces dernières ne pourraient plus être prorogées, on peut supposer que la nouvelle politique de la Région consisterait à favoriser l'ouverture de sites de plus petites dimensions. En faisant l'hypothèse qu'un petit site signifie une capacité moyenne ${ }^{3}$ de $145 \mathrm{kt} / \mathrm{an}$, il faudrait alors mettre en place 15 nouvelles ISDND d'ici $2027^{4}$ pour remplacer ces deux grandes installations $^{5}$. La situation est d'autant plus complexe que la plupart des ISDND franciliennes en sont à leur deuxième, voire troisième prolongation et doivent faire face à des oppositions locales (Mtibaa, Méry et Torre, 2009). Cela ne fait que rendre la création de nouveaux sites encore plus problématique, car les futurs riverains appréhendent la sédentarisation des installations, à l'image des expériences qui les entourent.

La situation de l'enfouissement est ainsi appelée à se complexifier, dans un contexte où la planification, réalisée à l'échelle des départements jusqu'en 2009, a conduit à un cloisonnement du territoire : un centre hyper productif en déchets, qui sont en partie stockés dans la Grande Couronne (disposant des superficies nécessaires à ce type d'installations). On se demande alors si un changement dans l'échelle de planification, tel qu'il a été décidé en 2004 et mis en œuvre fin 2009 (Boux et al., 2010), serait en mesure d'apporter des solutions à mettre en œuvre d'ici $2019^{6}$. En effet, avec les Plans Départementaux d'Elimination des Déchets Ménagers et Assimilés (PDEDMA), la vision du territoire francilien en matière de gestion des Déchets Ménagers et Assimilés (DMA) ${ }^{7} \mathrm{a}$ toujours été parcellaire. Dans un exercice d'analyse et de confrontation des anciens PDEDMA franciliens au Plan Régional d'Elimination des Déchets Ménagers et Assimilés (PREDMA) s'inscrivant dans une problématique plus générale de gouvernance territoriale et management public sur la question des échelles et périmètres pertinents de gestion, nous allons essayer de comprendre en quoi le changement de l'échelle de planification de 
la gestion des déchets est susceptible d'influencer la gouvernance en matière de stockage des déchets non dangereux.

\section{Les Plans Départementaux d'Elimination des Déchets Ménagers et Assimilés}

5 Nous commençons par faire le bilan des PDEDMA franciliens qui encadraient la gestion des DMA franciliens jusqu'à l'entrée en vigueur du PREDMA en novembre 2009, en nous focalisant sur les ISDND.

\subsection{Le département comme échelle de planification}

6 La notion de planification en matière de gestion des déchets date de la circulaire du 27 novembre 1969, qui invite les Préfets à établir des schémas départementaux de collecte et de traitement des DMA, et affiche l'objectif de "définir dans les meilleures conditions économiques possibles, les circuits de collecte, le nombre et l'implantation des dépôts et des usines, ainsi que leur capacité et rayon d'action ». Bien que ces schémas sans caractère obligatoire aient été peu appliqués, il s'agit d'une première étape pour la prise en compte sur une base territoriale $\mathrm{du}$ problème déchets (Rocher, 2006). La notion de planification territoriale de la gestion des déchets est réaffirmée avec la loi n $75-633$ du 15 juillet 1975 relative à l'élimination des déchets ${ }^{8}$, dont l'article 10 demande l'établissement de plans. Toutefois, le caractère obligatoire faisant toujours défaut, cette loi ne donnera lieu à la publication d'aucun plan (ADEME, 2004).

7 La loi 92-646 du 13 juillet 1992 rend obligatoire l'élaboration des PDEDMA et accorde trois ans aux départements pour les élaborer, affirmant ainsi la volonté du législateur de territorialiser la politique de gestion des déchets. Parmi les conditions particulières qui ont mis la problématique « déchets » à l'ordre du jour on relève la médiatisation durant la décennie 1980 d'affaires relatives à une gestion défaillante des déchets en France mais aussi en Europe ${ }^{9}$, la menace d'une pénurie en raison des difficultés d'implanter des exutoires $^{10}$ et la volonté des décideurs de restaurer un climat de confiance. Le PDEDMA est proposé par le législateur comme un moyen de contrôler à l'échelle d'un territoire la gestion des déchets.

8 Ainsi, dès les prémices de la planification territoriale de la gestion des DMA, le département s'impose comme échelle de planification. Ce choix est une sorte de "calquage sur les découpages administratifs existants, dans un souci de pertinence avec l'organisation déconcertée des services de l'Etat à qui est confiée la charge de l'élaboration des schémas» (Rocher, 2006) et ce pour une meilleure application du principe de proximité ${ }^{11}$. Il s'agit de superposer au mieux les espaces de production et d'élimination des déchets, dans un souci de réduction des impacts environnementaux de leur transport, qui est la base même du principe de proximité. En plus du caractère obligatoire de la réalisation des PDEDMA et des précisions apportées quant aux modalités de leur élaboration et contenu, la Loi du 13 juillet 1992 comporte une autre mesure phare, à savoir l'interdiction de l'enfouissement de déchets bruts à compter du $1^{\text {er }}$ juillet 2002: c'est dans ce contexte qu'apparait une notion importante pour l'activité des ISDND, celle de déchets ultimes. 


\subsection{Les PDEDMA franciliens}

9 Avant l'entrée en vigueur du PREDMA, l'île-de-France était couverte par 8 plans départementaux approuvés entre 2000 et 2006.

Tableau : Dates de publication et compétences d'élaboration des PDEDMA franciliens

\begin{tabular}{|l|c|c|}
\hline \multicolumn{1}{|c|}{ Département } & Date du plan & Compétence \\
\hline Seine-et-Marne (77) & $04 / 02 / 2004$ (révision plan du 09/09/1997) & Conseil Général \\
\hline Yvelines (78) & $15 / 03 / 2001$ (révision plan du 06/11/1995) & Préfecture \\
\hline Essonne (91) & $19 / 11 / 2002$ (révision plan de 29/09/1997) & Conseil Général \\
\hline Val d'Oise (95) & $\begin{array}{c}\text { 04/2006 (révision plan de 07/2002 qui est le } \\
\text { résultat de la révision du plan de 02/10/1996) }\end{array}$ & Conseil Général \\
\hline Paris (75) & $16 / 10 / 2001$ & Préfecture \\
\hline Hauts-de-Seine (92) & $28 / 06 / 2000$ (révision plan de 14/02/1997) & Préfecture \\
\hline $\begin{array}{l}\text { Seine-Saint-Denis } \\
\text { (93) }\end{array}$ & $26 / 07 / 2005$ (révision du plan de 24/03/1997) & Conseil Général \\
\hline Val-de-Marne (94) & $20 / 03 / 2003$ (révision plan de 22/08/1997) & Préfecture \\
\hline
\end{tabular}

Source : élaboration de R. Mtibaa

10 Comme le montre le tableau 1, excepté Paris, tous les départements franciliens en étaient à leur deuxième ou troisième plan ${ }^{12}$. Ces révisions sont la suite logique de l'évolution de la réglementation ainsi que des objectifs nationaux en matière de traitement des déchets. La directive européenne sur la valorisation des emballages ${ }^{13}$, qui s'est traduite à l'échelle nationale par le décret emballages ${ }^{14}$ a été le moteur de la révision des plans de première génération. Si la première génération a été l'œuvre des préfectures, la seconde est en partie celle des Conseils Généraux. C'est la loi relative aux libertés et responsabilités locales ${ }^{15}$ qui a prévu le transfert aux conseils généraux de la compétence d'élaboration des PDEDMA (Mallard et Barbier, 2006), et pour le cas de la région Île-de-France, au Conseil Régional.

\subsection{Définition des déchets ultimes}

11 La loi du 13 juillet 1992 définit la notion de déchets ultimes : est réputé ultime " un déchet, résultant ou non du traitement d'un déchet, qui n'est plus susceptible d'être traité dans les conditions techniques et économiques du moment, notamment par extraction de la part valorisable ou par réduction de son caractère polluant ou dangereux ${ }^{16}$. Cette grande flexibilité laissée dans la définition de ce qui est ultime est confirmée par la circulaire du 28 avril 1998 relative à la mise en œuvre des PDEDMA qui considère que la définition des déchets ultimes est : " propre à chaque périmètre d'élimination et découle directement du contenu et des objectifs du plan d'élimination proposé par chaque périmètre ».

Dans son bilan 2002 des PDEDMA, l'ADEME classe les définitions des déchets ultimes qui figurent dans les différents PDEDMA selon qu'elles sont claires ou déduites ${ }^{17}: 1$ ) une définition claire de ce qu'est un déchet ultime figure dans le plan ; 2) soit on déduit cette définition à partir des choix de gestion fait dans le plan. Dans le tableau ci-dessous nous appliquons cette classification aux définitions retenues dans les différents plans franciliens: 
Tableau : Définitions des déchets ultimes dans les PDEDMA franciliens

\begin{tabular}{|c|c|c|}
\hline Département & $\begin{array}{l}\text { Clarté de } \\
\text { définition }\end{array}$ & Définition \\
\hline Paris (75) & Aucune & \\
\hline $\begin{array}{l}\text { Hauts-de- } \\
\text { Seine (92) }\end{array}$ & Déduite & $\begin{array}{l}\text { Matériaux non incinérables et non valorisables en provenance de déchetteries, } \\
\text { d'unités de compostage de déchets verts- Fraction Fermentescible des Ordures } \\
\text { Ménagères (FFOM) et des centres de tri. }\end{array}$ \\
\hline int- & Déduite & $\begin{array}{l}\text { Mâchefers à forte fraction lixiviable et résidus d'épuration des fumées après } \\
\text { stabilisation. }\end{array}$ \\
\hline $\begin{array}{l}\text { Val-de- } \\
\text { Marne (94) }\end{array}$ & Déduite & $\begin{array}{l}\text { Résidus d'Epuration des Fumées d'Incinération d'Ordures Ménagères } \\
\text { (REFIOM), mâchefers non valorisables, matériaux non incinérables et } \\
\text { valorisables en provenance des déchetteries, unités de compostage de déchets } \\
\text { verts-FFOM et des centres de tri. }\end{array}$ \\
\hline $\begin{array}{l}\text { Seine-et- } \\
\text { Marne (77) }\end{array}$ & Claire & $\begin{array}{l}\text { - Ordures Ménagères Résiduelles (OMR): avant } 2007 \text { et du fait des déficits en } \\
\text { capacités d'incinération dans la Région. Après 2007, la Région (et notamment } \\
\text { Paris) devrait récupérer ses capacités d'incinération, les OMR ne seront alors } \\
\text { plus admis en ISDND. } \\
\text { - Encombrants: avant } 2007 \text { ils pourront être enfouis. Après 2007, passage } \\
\text { systématique en centre de tri. Le refus de tri sera considéré comme ultime. } \\
\text { - Déchets Industriels Banals (DIB) }{ }^{1} \text { : les bennes des entreprises produisant } \\
\text { moins de } 50 \mathrm{kt} / \text { an et les bennes des entreprises produisant plus de } 50 \mathrm{kt} / \mathrm{an} \text { à } \\
\text { condition qu'elles mettent en place un tri interne. } \\
\text { - Boues des stations d'épuration urbaines et industrielles stabilisées en cas de } \\
\text { difficultés d'épandage ou d'incinération. }\end{array}$ \\
\hline $\begin{array}{l}\text { Val d'Oise } \\
(95)\end{array}$ & Claire & $\begin{array}{l}\text { Les refus de tri, refus de tri-compostage, encombrants non valorisables, } \\
\text { mâchefers non valorisables, boues de stations d'épuration non valorisables en } \\
\text { agriculture et si le site d'enfouissement est équipé d'une installation de } \\
\text { valorisation du biogaz, les OMR stabilisées. }\end{array}$ \\
\hline $\begin{array}{l}\text { Yvel } \\
(78)\end{array}$ & laire & $\begin{array}{l}\text { Les REFIOM, les mâchefers non valorisables, les DIB, les déchets } \\
\text { d'assainissement, de démolition et les encombrants une fois extraite la part } \\
\text { valorisable. }\end{array}$ \\
\hline $\begin{array}{l}\text { Essonne } \\
(91)\end{array}$ & Déduite & $\begin{array}{l}\text { Les OM et encombrants qui sont ni recyclables, ni incinérables, les refus de tri } \\
\text { des DIB, les détournements de flux pendant les arrêts techniques et } \\
\text { défaillances des Unités d'Incinération d'Ordures Ménagères (UIOM), les } \\
\text { REFIOM et les mâchefers non valorisables. }\end{array}$ \\
\hline
\end{tabular}

Source : élaboration de R. Mtibaa

13 Cette synthèse appelle un ensemble de remarques. La première concerne la clarté de la définition: les départements de Paris et Petite Couronne ne donnent pas de définitions claires des déchets ultimes contrairement à ceux de la Grande Couronne. Ces derniers disposant d'ISDND sur leurs territoires, ils se doivent donc de préciser ce qui peut, ou pas, y être enfoui. La deuxième interroge la nature des déchets considérés comme ultimes: les déchets issus de l'incinération (résidus d'épuration des fumées d'incinération des ordures ménagères - REFIOM - et mâchefers non valorisables), les détournements de flux en périodes de maintenance ou défaillances techniques des UIOM et les refus de tri et de valorisation organique. L'enjeu de la prise en compte ou pas des DIB ouvre la question du chapitre de la complexité de la gestion des DIB, notamment pour les départements ne disposant pas de capacités d'enfouissement sur leur territoire. Bien que l'essentiel des déchets enfouis dans la région soient des DIB, ces derniers n'apparaissent pas toujours dans la liste des déchets considérés comme ultimes par certains départements. L'ADEME généralise ce constat à l'ensemble des PDEDMA en France (ADEME, 2004) et l'explique par le fait que ces derniers ne sont pas opposables aux entreprises. La prise en compte des DIB dans la gouvernance territoriale des déchets non dangereux et de leur stockage reste d'ailleurs un sujet à forts enjeux économiques (coûts pour les entreprises), sociaux et environnementaux (moindres "proximités" que les Déchets Ménagers et Assimilés - Méry, Mtibaa et Torre, 2009), que les documents de planification essaient d'intégrer autant que faire se peut-compte tenu des incertitudes sur les gisements (secret des affaires) et sur les déclinaisons réglementaires et territoriales du principe de proximité.

14 La région Île-de-France se présente ainsi comme un exemple représentatif de la flexibilité laissée par la circulaire du 28 avril 1998 relative à la mise en œuvre des PDEDMA quant à 
la définition des déchets ultimes. Mais une première analyse des plans départementaux montre également leur spécificité, puisque la définition des uns dépend des objectifs des PDEDMA des autres. Citons à ce propos le cas de la Seine-et-Marne dont la liste des déchets ultimes évolue dans le temps. En effet, au moment de la réalisation de son PDEDMA, certains incinérateurs du SYCTOM ${ }^{18}$ sont ou seront fermés pour mise aux normes européennes, d'où une importante déviation temporaire des flux vers le site de Claye-Souilly (77). Ainsi, le niveau de réflexion proposé par la circulaire ne s'entend pas au sens strict du département, mais le dépasse notamment par le biais de syndicats de traitement qui débordent les frontières départementales. Cela est d'autant plus vrai en Île-de-France, où la structure des capacités de traitement suit plus une logique radiale (Paris, Petite Couronne, Grande Couronne), qu'une logique départementale.

\section{Les PDEDMA franciliens et les flux interdépartementaux de déchets}

Nous allons maintenant nous intéresser à la façon dont les différents PDEDMA ont abordé la question des flux interdépartementaux de DMA. Il est à noter, comme le précise l'ADEME dans son bilan 2002 des PDEDMA (ADEME, 2004), que la région Île-de-France représente près de la moitié en tonnage des importations/exportations de déchets en France.

\subsection{Paris et la Petite Couronne}

Suite à la révision des PDEDMA des départements limitrophes ${ }^{19}$ et dans une volonté de coordination régionale, Paris a procédé en 2001 à la révision de son propre plan, afin que les autres départements puissent tenir compte de ses besoins en matière de traitements (Préfecture de Paris, 2001). Cette logique de planification est la conséquence d'une logique de terrain dans laquelle le SYCTOM, Syndicat intercommunal de traitement des ordures ménagères de l'agglomération parisienne, voit son territoire dépasser les frontières de Paris pour englober la quasi-totalité des communes des Hauts-de-Seine et de la Seine-Saint-Denis, ainsi que quelques communes du Val-de-Marne et des Yvelines. Parallèlement, l'essentiel de son appareil de traitement ${ }^{20}$ se trouve en-dehors de Paris, à la frontière des autres départements couverts par le Syndicat.

Le SYCTOM donne ainsi une légitimité aux transferts de déchets entre l'ensemble des départements franciliens. Il est la preuve qu'une logique de planification départementale perd totalement son sens comme le montrent les deux exemples suivants. Le premier est celui du Val-de-Marne. En 2003, trois incinérateurs traitaient une partie de ses DMA, le plus grand celui du SYCTOM (Ivry-sur-Seine (94)), réserve $500 \mathrm{kt} /$ an de ses capacités aux déchets venant de son secteur. Dans le même temps, le Val-de-Marne exporte ses déchets ultimes pour enfouissement notamment en Seine-et-Marne et appelle dans son PDEDMA à tenir compte de ses besoins: "une solidarité régionale, qui devra être prise en compte dans la révision du plan de la Seine-et-Marne, pour respecter le principe de proximité " (Préfecture de Paris, 2001). Le second concerne les Hauts-de-Seine. L'UIOM d'Issy-les-Moulineaux (92) appartient au SYCTOM et traite donc des déchets en provenance des Hauts-de-Seine, Paris et Yvelines. Selon le PDEDMA des Hauts-de-Seine la production totale de DMA du SYCTOM étant supérieure à ses capacités d'incinération, une partie des déchets pesés sur ses UIOM est transférée par la suite vers les ISDND du Plessis-Gassot (95) et de Claye-Souilly (77). 

dans les plans rien ne laisse suspecter d'éventuelles tensions entre les départements ou d'agitation dans le monde associatif local quant au fait que les déchets traités sur certaines installations viennent d'autres départements. Au contraire, les autorités en charge de la réalisation des PDEDMA essaient de tenir compte de la complexité du territoire francilien en matière de gestion des DMA dans leurs prévisions. C'est dans cette logique que le département du Val-de-Marne se fixait comme objectif de « mieux inscrire la gestion des déchets du Val-de-Marne dans une réflexion régionale " (Préfecture Val-de-Marne, 2003) le justifiant par une solidarité interdépartementale qui s'opère dans les deux sens ${ }^{21}$.

\subsection{La Grande Couronne}

L'intégration de la dimension régionale dans la planification départementale s'est également retrouvée dans les plans des départements de la Grande Couronne, d'autant plus qu'ils disposent de capacités d'enfouissement utilisées par toute la région.

\section{Les Yvelines}

Les orientations prises par le PDEDMA des Yvelines sont influencées par une situation de surcapacité en matière d'incinération qui pose la question de la viabilité des installations, suite à la mise en place des collectes sélectives. Le plan des Yvelines préconisait ainsi une meilleure répartition des déchets entre les équipements existants à l'échelle régionale, voire interrégionale de sorte à assurer le plein emploi des capacités existantes (Préfecture Yvelines, 2001). Il proposait également de procéder à un transfert entre filières (enfouissement et incinération), en partant du principe qu'à partir de juillet 2002 les déchets non ultimes ne pourraient plus aller en ISDND.

21 Mais ce plan n'a pas tenu compte des déchets industriels banaux (DIB) qui constituent la part la plus importante des déchets enfouis, estimant qu'il est difficile de prévoir l'ampleur des transferts du fait de la méconnaissance de ces déchets (quantités, composition...). La préfecture conclue alors que les collectivités ne doivent pas surdimensionner leurs capacités de traitement en l'absence de garanties sur les quantités à traiter. Un bilan des capacités/besoins en enfouissement a tout de même été réalisé et a révélé que les capacités de stockage annuelles autorisées étaient atteintes sur les deux ISDND et que le département ne disposait au moment de sa réalisation d'aucune capacité résiduelle d'accueil. D'autre part, il évaluait à $100 \mathrm{kt} / \mathrm{an}$ les capacités d'enfouissement à créer sur le département ${ }^{22}$ avant 2005.

\section{L'Essonne}

Lors de la révision de son plan en 2002, le Conseil Général de l'Essonne a rappelé que du fait de son appartenance à la Grande Couronne, il devait tenir compte des objectifs des départements limitrophes. Il préconisait ainsi une approche complète des flux interdépartementaux des déchets, pour éviter de sur-dimensionner ses installations. Ce bilan a révélé que des OMR des autres départements sont incinérés sur les installations essonniennes, alors que $15 \%$ des OMR essonniennes sont enfouies en dehors du département (Hanche (28) et Soignolles-en-Brie (78)). De plus, l'Essonne importe (pour tri/incinération) plus de DIB qu'elle n'en exporte (pour enfouissement).

Développement durable et territoires, Vol. 3, n 3 | Décembre 2012 


\section{La Seine et Marne et le Val d'Oise}

23 Ces deux départements ont la particularité d'être une réserve de capacités d'enfouissement pour toute la région. Un rôle qui est assumé dans les deux plans et à mettre en regard des discours d'élus et d'acteurs associatifs sur le statut de «poubelle régionale ». Les plans traduisent une certaine résignation, ainsi qu'une conscience de la marge de manœuvre quasi inexistante dans l'immédiat pour rééquilibrer les capacités de stockage à l'échelle régionale.

Le plan de la Seine-et-Marne réaffirme de manière explicite son engagement en termes de solidarité régionale pour ce qui est de l'enfouissement des DMA, tout en prévoyant une réduction : "pour préserver l'avenir de ces sites » (Conseil Général Seine-et-Marne, 2004), prenant ainsi acte du caractère épuisable de la ressource naturelle spatiale déjà mise en avant par les économistes dans le domaine du stockage des déchets (Bertolini, 2003; Ready et Ready, 1995 ; Chang et Schuler,1991; Lund, 1990).

La Seine-et-Marne démontre la complexité de l'échelle territoriale de gestion des DMA en Île-de-France quand elle procède à des recommandations régionales dans son plan départemental: 1) Le SYCTOM devrait réduire de $400 \mathrm{kt} / \mathrm{an}$ ses apports de DMA; Les collectivités hors SYCTOM devraient réduire de $310 \mathrm{kt} / \mathrm{an}$ leurs apports de DMA; Les entreprises extérieures à la Seine-et-Marne devraient réduire de $220 \mathrm{kt} / \mathrm{an}$ leurs apports de DIB ; Création de nouvelles capacités de traitement dans les autres départements.

Le département du Val d'Oise s'inscrit dans la même logique et décrit ses ISDND comme: « des outils à préserver y compris dans une perspective régionale » (Conseil Général Val-d'Oise, 2006).

La flexibilité dans la réception des déchets des autres départements franciliens sur les deux grands ISDND du Val d'Oise et de la Seine-et-Marne est une garantie du bon fonctionnement du système francilien de gestion des déchets en cas de problèmes majeurs. L'enfouissement étant perçu comme une assurance de résilience pour l'ensemble du système de gestion des déchets à l'échelle du territoire régional, on n'en prévoit que la baisse au vu de tous les efforts faits en amont, alors que les départements de la Seine-etMarne et du Val-d'Oise, qui détiennent la plus grande capacité de stockage, mettent l'accent sur la nécessité de préserver ces sites du fait du rôle qu'ils jouent. Ainsi, au nom de la solidarité régionale, l'enfouissement des déchets ultime remonte la problématique de la gestion des déchets de l'échelle départementale à l'échelle régionale, longtemps avant la réalisation du PREDMA.

\section{Le Plan Régional d'Elimination des Déchets Ménagers et Assimilés}

Le 2 mars 1982 a été promulguée la «loi relative aux droits et libertés des communes, des départements et des régions", point de départ d'une grande réforme institutionnelle qui a placé les Conseils Généraux au cœur du paysage institutionnel français. En matière de gestion des DMA, cette politique se traduit par la loi de décentralisation n²004-809 du 13 août 2004 relative aux libertés et responsabilités locales qui va transférer la responsabilité d'élaboration du PEDMA aux Présidents des Conseils Généraux (Mallard et Barbier, 2006), et pour l'île-de-France, au président du Conseil Régional, renforçant ainsi son rôle dans le développement stratégique de son territoire. 


\subsection{De la loi de décentralisation au PREDMA} décentralisation a été enclenché, au même titre que la réalisation des SDRIF (Schéma Directeur de la Région île-de-France), SRDE (Schéma Régional de Développement Economique), PDU (Plan de déplacement Urbain) et PRQA (Plan Régional de Qualité de l'Air).

Précisons que si, dans la loi, les premières compétences régionales en matière de gestion des déchets ont vu le jour à partir de 2002 ${ }^{23}$, dans les faits c'est depuis 1992 que le Conseil Régional s'est penché sur la question et ce par la mise en œuvre des contrats Terre Vives (CRIdF, 2007). Cet intérêt avait pour ambition, d'une part d'accélérer le processus de réalisation des équipements nécessaires à la Région pour respecter l'échéance de $1^{\text {er }}$ juillet $2002^{24}$, et d'autre part de donner aux collectivités de la Région des objectifs de collectes séparatives et de valorisation conformes aux orientations de la circulaire Voynet d'avril 1998 (CRIdF, 2007). Ainsi, alors que la Région jouait déjà un rôle important en matière de gestion des DMA franciliens, ce n'est qu'à partir du 13 août 2004 que ce rôle est institutionnalisé par la loi de décentralisation. Doté des pouvoirs de planification sur l'ensemble des DMA et du territoire, le CRIdF se lance dans l'exercice d'élaboration du PREDMA le 8 juin 2006 lors de la première réunion de la commission consultative de ce plan. Nous allons maintenant analyser le PREDMA en partant des trois années d'élaboration qui ont donné lieu à une multitude de travaux, pour aller vers une présentation synthétique des décisions liés aux ISDND dans le plan adopté le 26 novembre 2009. Comme pour les PDEDMA, nous nous intéresserons à la question des flux interdépartementaux et de la position des différents acteurs sur la question de l'enfouissement des déchets ultimes. Ceci nous permettra de confronter les arguments débattus pendant la phase d'élaboration avec ceux retenus dans le plan.

\subsection{L'élaboration du PREDMA}

31 Le législateur a confié à la Région Île-de-France la responsabilité de planifier la gestion des DMA. Pour réussir cette mission le CRIdF s'est doté des moyens nécessaires pour mener une large concertation associant le plus grand nombre d'acteurs publics et privés. Cette démarche de concertation est considérée comme une des trois conditions de réussite de tout exercice de planification dans le domaine de la gestion des DMA, à savoir: «La qualité du processus d'échange et de négociation entre acteurs; le réalisme (perçu par les élus) du cadrage technique préconisé; la crédibilité de l'autorité pilote du plan, notamment quant à sa capacité à faire respecter les orientations prises» (Barbier et Mallard, 2006). Différentes instances ont ainsi été mises en place pour donner à l'ensemble des acteurs l'occasion de participer à la construction du plan (commission consultative, comité de coordination, 7 groupes de travail thématiques ${ }^{25}$ ).

En plus de ce dispositif de travail, des occasions ont été créées pour permettre à l'ensemble des acteurs d'échanger et d'avoir une vision globale du travail réalisé au niveau de chaque instance ${ }^{26}$. Finalement, les franciliens ont été également sollicités dans le cadre de groupes de discussion et d'une conférence de citoyens, la première en France qui ait été appliquée à la gestion des déchets. Ainsi le CRIdF a investi dans différents outils de concertation (en particulier pour près de $200 \mathrm{k} €$ de sous-traitance à des médiateurs 
environnementaux expérimentés) tout en faisant preuve d'innovation dans le choix des méthodes et se démarque ainsi de ses prédécesseurs qui ont mobilisé peu d'outils méthodologiques spécifiques pour assurer une concertation de qualité.

\section{Les positions des différents acteurs} Région Île-de-France, le choix de passer à un plan régional va permettre d'assurer une cohérence du service de gestion des déchets sur l'ensemble du territoire et une meilleure application du principe de proximité. Le groupe des élus socialistes déclare ainsi: « La densification de l'hypercentre de notre agglomération engendre une répartition inégale des déchets sur l'ensemble du territoire régional et donc des flux importants du cœur aux franges de l'île-deFrance. Les objectifs du PREDMA montrent combien l'échelon régional est pertinent pour l'élaboration d'une stratégie globale du tri et du traitement des déchets et tendent vers une organisation des flux de gestion des déchets » (CRIdF, 2007). l'inégalité spatiale dans la région au nom de la solidarité régionale. Les Conseils Généraux du Val d'Oise et de la Seine-et-Marne précisent à ce propos que, du fait d'une situation géographique particulière, leurs départements supportent déjà une forte concentration d'installations de traitement des déchets (notamment des ISDND) qui bénéficient à toute la région, rappelant ainsi l'application du principe de proximité sur leurs territoires respectifs bien avant le PREDMA. Estimant que « la solidarité régionale ne peut pas continuer à s'organiser au détriment de la santé et du cadre de vie de citoyens déjà concernés par la présence d'ISDND ou d'incinérateurs ", ils attendent du PREDMA un rééquilibrage des capacités de traitements visant à diminuer leur concentration dans certains départements, ainsi qu'une nouvelle approche des besoins en équipements avec une meilleure répartition du coût environnemental lié à ce type d'installations.

(A tranchés à l'égard du plan. L'Associon Pour le Plateau Agricole de Centre Essonne (APPACE) s'inquiète de voir les installations de traitement de déchets se créer au détriment des terres agricoles et appelle à mettre un frein au: "gigantisme des structures de traitement des déchets et d'arrêter de considérer les espaces agricole péri-urbains comme des espaces vides", alors que la Confédération des 
Associations de Protection de l'Environnement de Fontainebleau et Alentours (CAPEFA) suggère que, si la gestion des décharges est déléguée aux opérateurs privés, il est nécessaire de maintenir une certaine maîtrise du foncier pour ne pas s'enfermer dans des opportunités foncières purement spéculatives. Le contrôle doit être aux mains de la collectivité qui reçoit l'installation, de sorte qu'elle puisse exiger dans le cahier de charges de sa régie l'intégration paysagère qu'elle souhaite pour l'installation en question.

La Fédération Nationale des Activités de la Dépollution et de l'Environnement (FNADE) plaide pour des installations de traitement des déchets tel que les ISDND de grande taille, afin d'éviter la dilution des outils de petite taille et pour une meilleure performance économique et environnementale (optimiser la valorisation matière et énergétique). Elle estime que du fait de la politique des $3 \mathrm{R}^{27}$ : « on s'attend à une baisse des tonnages incinérés et enfouis, il est donc nécessaire d'élargir les bassins versants des installations existantes de sorte à les saturer et par la même occasion favoriser la production d'énergie renouvelable ». Le principe de proximité ne doit pas être un frein mais être assoupli en cas de présence de mode de transport alternatif permettant de desservir les installations existantes.

L'ensemble des acteurs semble s'accorder sur le fait que le PREDMA est l'occasion de favoriser le report modal et que l'on doit tenir compte de la possibilité de développer un acheminement alternatif des déchets dans les critères de choix de localisation des installations. Il en va de même pour la nécessité d'améliorer la connaissance des DIB (quantité et composition) traités dans les mêmes installations que les DMA. Cet état des lieux conditionne la qualité de la prospective quant aux capacités de traitement à prévoir et notamment pour les ISDND.

40 Finalement, un appel est fait par l'ensemble des acteurs à la Région pour qu'elle se penche sur le phénomène Not In My Back Yard qui bloque tout nouveau projet d'installation. Le Groupe des élus Communistes, Alternative Citoyenne et Républicain du Conseil Régional d'Île-de-France (CACR) propose d'engager des conférences de citoyens en amont pour faire valoir l'intérêt général, cesser de mettre les populations devant le fait accompli et décider avec elles des lieux d'implantation des équipements.

41 Ainsi, l'accord de l'ensemble des acteurs sur le changement d'échelle de planification pour la Région Île-de-France n'exclut pas certaines inquiétudes quant à l'équité spatiale, sujet qui concerne de près les ISDND. Certains s'inquiètent de voir le changement d'échelle de planification rendre légitime, voire renforcer des spécialisations territoriales perçues localement comme injustes. D'autres insistent au contraire sur la non dispersion des installations et l'agrandissement de leur bassin versant. L'occupation de l'espace et la concurrence entre les usages en milieu agricole touchent aussi de près les ISDND, gourmandes en superficie et souvent implantées en milieu rural. Les questions d'équité spatiale, de transport des déchets et de leurs natures domineront l'ensemble du discours concernant la planification du stockage des déchets. Enfin, la prise en compte des phénomènes qualifiés de NIMBY constitue une nouveauté : la pénurie de capacités de traitement dans la région menace toujours et dans le même temps des projets n'aboutissent pas en raison des oppositions locales. 


\section{La planification du stockage à l'échelle régionale}

\section{Equité spatiale}

Dès le début de la concertation, les autorités en charge de la réalisation du PREDMA pointent la question de la répartition géographique des unités de traitement et de stockage, en estimant que «le sentiment d'inégalité face à cette problématique doit être levé, les flux de déchets ne doivent plus voyager dans un seul sens » (CRIdF, 2007). Elles appellent ainsi à tenir compte de la dimension sociale des installations, sujet peu abordé jusque-là par les PDEDMA. Cette orientation est confirmée par M. Vampouille (CRIdF, 2008) alors viceprésident de la Région chargé de l'environnement, qui résume comme suit la position du CRIdF: « Tous les secteurs de la Grande Couronne s'estiment être la poubelle de l'Île-de-France, et notamment de Paris. Le Val d'Oise est persuadé d'être la poubelle de Paris. La Seine-et-Marne est persuadée d'être la poubelle de Paris. Cette dimension conflictuelle est très présente dans l'imaginaire. L'un des enjeux du plan est de faire en sorte que ce conflit puisse se résorber. Il restera des déchets à enfouir, indépendamment de l'objectif de réduction chiffré que nous retiendrons. Il est peu probable d'envisager la construction d'un centre d'enfouissement sous les champs Elysées. Par contre, il n'est pas complètement impossible que les déchets fassent un chemin inverse: des zones de méthanisation pourraient être installées un peu plus prés de la zone centralisée. Celles-ci pourraient alors recevoir des déchets organiques provenant des villes de la grande couronne ».

Cette position est représentative d'une façon de voir le territoire comme un ensemble et non comme une superposition de syndicats de traitement. On passe d'un groupe de PDEDMA, qui se prononçait sur la question en fonction de son appartenance à la Petite ou la Grande Couronne, sans vraiment se préoccuper des questions d'équité spatiale, à un seul plan et une concertation avec tous les acteurs pour une réponse commune qui devrait mieux tenir compte des injustices locales. En plus de la question des flux interdépartementaux, le CRIdF met d'entrée en cause la taille des installations de traitement des déchets: "Concentrer des déchets d'un périmètre large sur des installations centrales de capacités très importantes peut contribuer à alimenter des phénomènes de rejets des riverains, mécontents de constater que des déchets venants de loin convergent à proximité de chez eux» (CRIdF, 2007), soulignant ainsi des dynamiques de proximité typiques de la gestion des déchets (Méry, Mtibaa et Torre, 2009). Alors que les PDEDMA se sont abstenus jusquelà de tout commentaire sur les questions d'acceptabilité des installations et se sont penchés sur les flux pour mieux les cerner, le PREDMA montre qu'en changeant l'échelle de planification on tient compte d'un nombre plus grand d'acteurs. Ce qui oblige à tenir compte des préoccupations locales sur la table de la planification d'un territoire plus grand. Dans d'autres champs d'application de l'environnement comme la gestion des bassins-versants hydrologiques, ce genre d'élargissement a même été vu comme une souplesse accrue en matière de compensations territoriales, en permettant des négociations plus équilibrées et donc plus acceptables (Fischhendler et Feitelson, 2003).

\section{Choix de localisation}

«La Région a la responsabilité de la planification. Cependant, la Région n'a pas de suprématie sur les départements ou les communes et ne pourra pas imposer d'équipements sur un lieu précis » (ORDIF, 2008). Ainsi, bien que le CRIdF soit l'institution qui est le plus au fait des flux de transport que génère la gestion des DMA dans la région, elle peut au mieux faire des préconisations, car le plan n'a pas de rôle prescriptif. Nous sommes dans une situation où 
"la Région peut interdire ce qui n'est pas dans le plan, mais ne peut en aucun cas l'imposer" (ORDIF, 2008). Mais les acteurs en charge de la réalisation du plan ne peuvent se limiter à ce constat. En effet, le code de l'environnement stipule dans son article L541-14 que le plan a l'obligation de dresser l'inventaire des installations nécessaire pour la réalisation de ses objectifs et de définir les critères de leur localisation. Dans le même article, une mention spécifique est faite concernant la planification de nouvelles ISDND : « Le plan doit prévoir obligatoirement, parmi les priorités qu'il retient, des centres de stockage de déchets ultimes issus du traitement des déchets ménagers et assimilés ${ }^{28}$. Cette mention particulière montre l'importance aux yeux du législateur des ISDND comme installations permettant de sécuriser l'ensemble du système de gestion des DMA sur un territoire. Ainsi, le CRIdF a mené un exercice de consultation spécifique des différents acteurs concernés sur le thème des conditions d'implantation et de pérennisation des installations.

Trois catégories de critères de localisation ont été traités comme suit :

Thème 1 : La gouvernance, la concertation, la transparence de l'information et le suivi des installations

\section{Source: CRIdF, 2008}

De cette première proposition on retiendra que la clé pour l'implantation de toute nouvelle installation c'est la transparence de l'information, à tous les niveaux du processus d'implantation (recherche de site, expertises, décision), et de sa pérennisation (suivi du fonctionnement de l'installation).

Thème 2 : La recherche de Cohérence, d'efficacité des installations et d'amélioration des techniques utilisées

Source : CRIdF, 2008

Deux idées sont à retenir : la première correspond au désir de l'ensemble des acteurs de voir les nouvelles installations s'inscrire dans une approche développement durable en cherchant à faire jouer des synergies entre les installations et l'environnement dans lequel elles s'inscrivent ${ }^{29}$; la deuxième reflète une certaine appréhension vis-à-vis du gigantisme industriel, appréhension dont la conscience a déjà été traduite dans le passé par différents auteurs (Schumacher, 1973). Il faut préciser que certaines associations de lutte contre des projets actuels d'ISDND en Île-de-France étaient fortement représentées parmi les intervenants de l'atelier. Les critères de localisation qu'elles proposent sont en relation directe avec leurs inquiétudes et rappellent le discours qu'elles portent contre ces nouvelles installations : pourquoi créer de nouvelles installations sachant que, si l'on tient compte des objectifs de réduction de déchets, il ne doit pas rester beaucoup de DMA à enfouir, pratiquant ainsi une montée en généralité vers l'amont de la filière désormais classique dans les argumentations autour de projets controversés. Dans le discours ces associations on dénote aussi la peur de voir ces installations, une fois implantées, s'agrandir par leurs tailles, leurs capacités et dans le temps. D'où la proposition de faire attention à ne pas créer des installations surdimensionnées ne prenant en compte ni les évolutions technologiques, ni les efforts de prévention engagés.

Thème 3 : La proximité

Source : CRIdF, 2008 
critère d'implantation ne concerne pas l'installation au sens strict mais se présente comme un élément d'un système qu'il faut optimiser en faisant jouer la proximité amontaval. L'ISDND, devrait justifier sa localisation et ses capacités en fonction des gisements qu'elle va drainer et de la localisation de ces derniers, mais cette recommandation est mise à mal lorsqu'on tient compte des DIB pour lesquels on ne dispose pas de ce type d'informations.

\section{DIB et exercice de planification}

Alors que les PDEDMA ne tenaient pas tous compte des DIB lors de l'exercice de planification, le PREDMA tranche la question en recommandant la prise en compte de ces déchets tout au long du processus de planification ${ }^{30}$ pour : «juger la réelle adéquation entre le gisement et les capacités de traitement des déchets non dangereux» (CRIdF, 2007). Toutefois, cette bonne volonté fait face à la méconnaissance des quantités de DIB produites et traitées dans la région. Il n'existe au moment d'élaboration du PREDMA aucune enquête exhaustive publique quant aux quantités de DIB exactes traités sur les installations franciliennes. Les différents acteurs souligneront ce problème tout au long de la réalisation du PREDMA et notamment lorsqu'il s'agit des ISDND, car les DIB utilisent la plus grande partie des capacités de stockage disponibles dans la Région, généralement redevable de contrats privés. Cette situation est insupportable aux yeux de certains acteurs, qui peuvent admettre que l'on héberge sur leur territoire une installation devant servir l'intérêt général mais deviennent nettement moins compréhensifs si ces installations sont destinées à des entreprises privées.

\subsection{L'adoption du PREDMA}

\section{Définition des déchets ultimes}

Le PREDMA a retenu deux définitions pour les déchets ultimes, une spécifique aux DMA et l'autre aux DIB.

Définition du « déchet ultime » pour les déchets ménagers et assimilés :

51 «A l'horizon 2014, aucun déchet brut ne peut être directement enfoui.

Les déchets ménagers pouvant être enfouis à l'horizon 2014 sont :

- les refus de tri de collecte sélective emballages et journaux refus magazines

- les refus de tri-compostage sur OMR (refus des opérations de préparation des ordures résiduelles en vue de l'extraction de leur fraction fermentescible);

- les encombrants non valorisables après tri ;

- les déchets ultimes à caractère exceptionnel (mâchefers non-conformes à la circulaire du 9/05/94 ; les boues, composts/ digestats non-conformes aux normes en vigueur) ;

- les déchets résultants de situations exceptionnelles (incidents sur des installations).

A l'horizon 2019, les refus de tri de collecte sélective emballages et journaux refus magazines ne sont plus considérés comme des déchets ultimes.

Par rapport à la situation 2005, ne seront plus acceptés à l'enfouissement les OMR ainsi que les refus de tri des collectes sélectives emballages ménagers et journaux revues magazines. » (CRIdF, 2009) 
Définition du « déchet ultime » pour les déchets des activités

作 à une définition des déchets ultimes. Comme déjà évoqué plus haut, ce n'est pas un hasard si les définitions les plus précises viennent de ce département. S'est également posée la question d'une définition locale plutôt que régionale des déchets ultimes, afin de respecter les spécificités territoriales. Comme mentionné dans les encadrés ci-dessus. ce choix (fondamental dans toute gouvernance territoriale des déchets car caractérisant les exutoires finaux et contraignant tous les flux amont), s'est porté sur une définition régionale, traduisant une solidarité régionale et une forte légitimation de la gouvernance à l'échelle régionale. La nécessité d'une cohérence régionale a primé sur les velléités de souveraineté départementale. Il est enfin intéressant de noter que si catégorisation il y a eu, c'est entre l'origine institutionnelle des déchets : DMA d'une part, DIB d'autre part, ce qui rejoint le constat d'une forte différence de connaissance et de gouvernance entre ces deux types de déchets.

\section{Objectifs retenus}

Le plan a estimé comme suit les besoins de capacités d'enfouissement pour les déchets ménagers et assimilés ainsi que pour les déchets des activités à l'horizon de 2014 et 2019:

Tableau : Les besoins de capacités en ISDND pour les déchets franciliens

\begin{tabular}{|l|c|c|}
\hline & 2014 & 2019 \\
\hline $\begin{array}{l}\text { Tonnage francilien prévisionnel de déchets } \\
\text { ménagers et assimilés en ISDND }\end{array}$ & $567514 \mathrm{t}$ & $623165 \mathrm{t}$ \\
\hline $\begin{array}{l}\text { Tonnage francilien prévisionnel de déchets } \\
\text { d'activités en ISDND }\end{array}$ & $1872637 \mathrm{t}$ & $1796822 \mathrm{t}$ \\
\hline $\begin{array}{l}\text { Besoin prévisionnel de capacité en ISDND pour } \\
\text { des déchets franciliens }\end{array}$ & $2440151 \mathrm{t}$ & $2419987 \mathrm{t}$ \\
\hline
\end{tabular}

Source: CRIdF, 2009

Une estimation des besoins en capacités a également été réalisée par le CRIdF sur la base des dates de fermetures des ISDND inscrites dans les arrêtés, ainsi que les besoins en enfouissement pour les années 2005, 2014, 2019 et 2024. Cette estimation est faite avec comme hypothèse l'absence d'ouverture de nouvelles ISDND: 
Figure : Evolution des capacités d'enfouissement (hors projets) au regarde de l'estimation des besoins d'enfouissement aux horizons 2014, 2019 et 2014

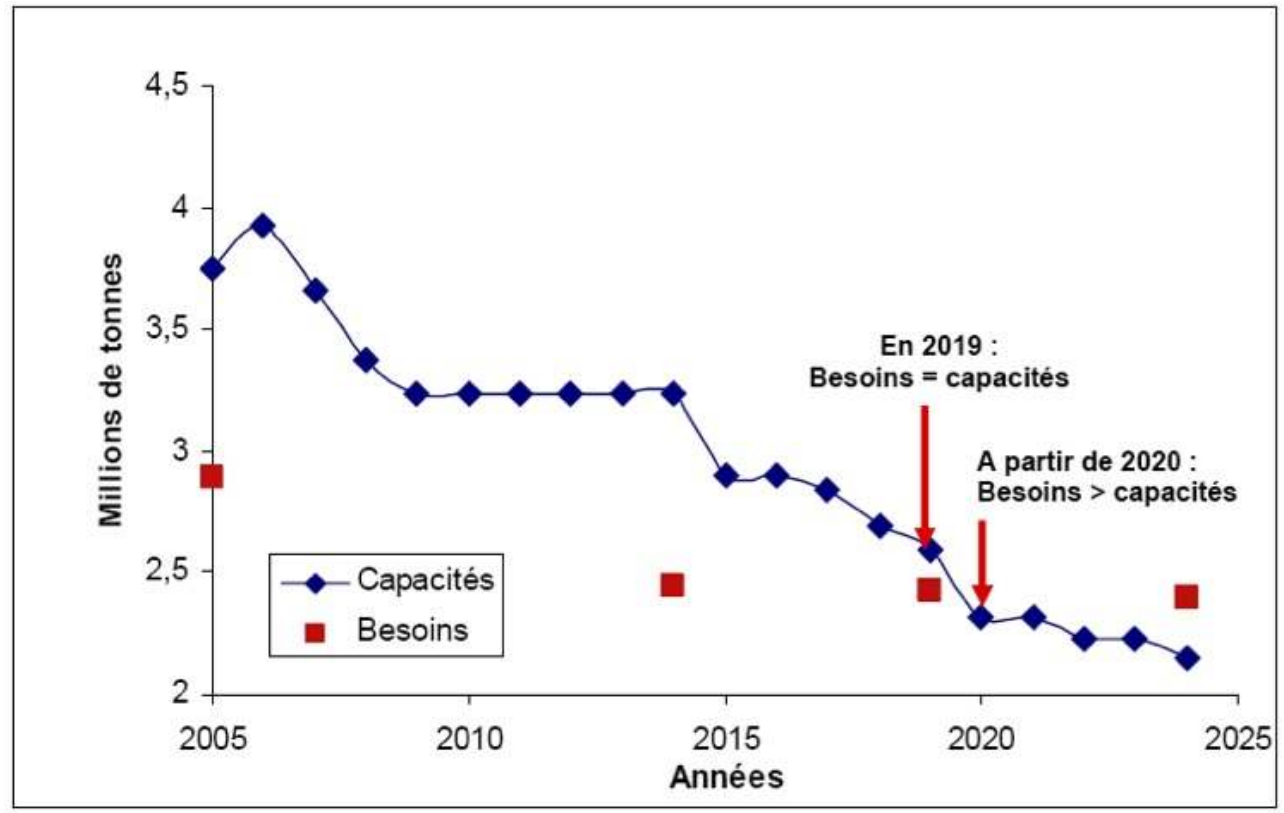

Source: CRIdF, 2009 des objectifs fixés par le PREDMA en matière de prévention, valorisation des DMA et de réduction de l'enfouissement au profit de la valorisation matière et énergétique des DIB. Cela dit, en l'absence de nouveaux projets et avec l'arrivé à fin d'exploitation de certaines ISDND, la situation de pénurie pourrait se faire sentir dans la Région dès 2020. Les capacités vont continuer à être géographiquement concentrées en Seine-et-Marne et dans le Val-d'Oise avec des contributions respectives de l'ordre de 52 et $48 \%$ des capacités totales de stockage dans la Région à l'horizon 2019.

base de cette prospective, un principe de rééquilibrage territorial a été retenu comme suit: «La création de nouvelles capacités sera appréciée de manière à assurer un rééquilibrage territorial à l'ouest et au sud de l'Ile de France, notamment pour faire face aux fermetures de sites dès 2020 et aux incertitudes des projections, en particulier sur les déchets des activités économiques. Ainsi, aucun projet d'extension ou de création de capacités ne devra être prévu dans les départements du Val d'Oise et de Seine et Marne jusqu'en 2019» (CRIdF, 2009).

Compte tenu des éléments exposés au paragraphe 3.2.2, les incertitudes sur les flux futurs de déchets d'activités économiques et la limitation de l'échéance temporelle de la prospective du plan à 2019 certains départements sont inquiets. Ainsi, le Val d'Oise précise : "au regard des projets de nouvelles capacités d'enfouissement déposés dans les préfectures d'Ile-de-France, la disposition du plan interdisant la création de nouvelles capacités d'enfouissement dans notre département ne changera pas de manière significative la situation actuelle, les installations de stockage de déchets aujourd'hui exploitées dans le Val d'Oise ayant des autorisations d'exploiter jusqu'en 2027" (Conseil Général Val d'Oise, 2009). La Seine et Marne expose quant à elle : "une définition de seuils maximum de capacité ou de durée d'exploitation est indispensable pour lutter contre le phénomène subi de concentration et d'extension indéfinie" (Conseil Général Seine et Marne, 2009).

Développement durable et territoires, Vol. 3, n 3 | Décembre 2012 


\section{Conclusion}

francilien, vue sous l'angle du changement d'échelle de gouvernance appliqué au stockage des déchets non dangereux. Partir de huit plans départementaux pour arriver au PREDMA, avec une meilleure cohérence spatiale et temporelle du stockage des déchets, pouvait passer pour une gageure. Et en effet, rares sont les départements qui se sont lancés dans un tel exercice de prospective quant à l'adéquation des besoins et des capacités futures, probablement parce que les plans avaient une portée départementale alors que les besoins de stockage sont d'ampleur régionale. Mais le PREDMA a fini par donner une vision régionale d'une activité qui l'a toujours été et a fourni une prospective qui dépasse l'échelle temporelle du plan ${ }^{31}$. De plus, la légitimation d'une échelle régionale de gestion via un déchet ultime défini régionalement et non plus par département induit nécessairement une plus forte proximité organisationnelle entre acteurs des territoires franciliens, ce qui peut au moins contribuer à diminuer les coûts de transaction, inévitables dans tout processus de gouvernance territoriale (Casteigts, 2009)

Cela dit, si cette démarche permet d'envisager plus clairement les limites des capacités disponibles dans la région Île-de-France, elle n'apporte pas de recommandations fortes au vu de la situation de pénurie future. En effet, interdire tout projet de nouvelle installation ou d'extension sur site existant dans le Val-d'Oise et la Seine-et-Marne jusqu'à 2019 n'a pas vraiment un grand impact vu que les deux sites en question (Claye-Souilly (77) et Le Plessis-Gassot (95)) sont autorisés jusqu'à 2026 et 2027. Autrement dit, jusqu'à cet horizon, ces deux départements constitueront la réserve de la région en capacités d'enfouissement. Cette décision ne fait que reporter le débat du risque de pénurie de capacités de stockage à 2019, année de révision du PREDMA.

61 Le changement dans l'échelle de planification a ainsi permis de faire le point sur la situation globale de l'enfouissement mais pas de résoudre la question de la taille et de la localisation de futures ISDND. Ce constat est confirmé par les Conseils Généraux du Val d'Oise et de Seine-et-Marne qui soulignent la non adéquation entre les besoins et les autorisations accordées d'ici 2019 et les ambiguïtés sur le rééquilibrage territorial au-delà de cette échéance. Ainsi, le PREDMA n'a fait que rendre légitime d'un point de vue institutionnel la situation actuelle du stockage dans la région. Le changement d'échelle de planification pour le cas particulier des ISDND se traduit par un simple réajustement du secteur couvert par le plan à la situation réelle du terrain.

62

Enfin, le PREDMA, en ne se prononçant pas sur les conflits de localisation, s'inscrit dans la tradition des PDEDMA qui ont omis de remonter les informations quant aux tensions locales subies lors de projets de nouvelles ISDND ou d'extensions sur d'anciens sites (ADEME, 2010). Que ce soit au cours des réunions, dans les documents de travail, ou dans le plan final, à aucun moment les instances du plan ne se sont prononcées sur ces projets de manière explicite, alors que les associations et les porteurs de projets étaient présents et sont fortement intervenus tout au long du processus d'élaboration. Le PREDMA reste un document de planification qui essaye de concilier tous les acteurs, conciliation devenue encore plus difficile avec le changement d'échelle de planification. En témoigne la formulation de la première réserve émise le 18 novembre 2009 par l'avis de la commission d'enquête publique du PREDMA, qui dans un souci de clarté formule les préconisations du PRDEMA en matière d'extensions ou de nouvelles capacités 
d'enfouissement comme suit: "si toute nouvelle capacité, ou extension de capacité d'enfouissement est interdite pendant la durée du plan, dans le Val d'Oise et en Seine et Marne, le plan n'interdit aucunement de nouvelles capacités ou extension de capacité dans les départements de l'Essonne et des Yvelines même si cela conduit à des capacités qui seraient supérieures aux besoins exprimés tant que cela conduit à un rééquilibrage géographique des capacités d'enfouissement sur la Région ».

\section{BIBLIOGRAPHIE}

ADEME, 2004, Plans départementaux d'élimination des déchets ménagers et assimilés - Bilan 2002, Angers, $102 \mathrm{p}$.

ADEME, 2007, Pénurie d'exutoires pour les déchets résiduels - Nouvelle approche ADEME et résultats, Angers, $82 \mathrm{p}$.

ADEME, 2010, Analyse géographique et sociale du stockage des déchets non dangereux en France : état des lieux en 2010, $108 \mathrm{p}$.

Barbier R., Mallard P. 2006, "Planification de la gestion des déchets: quelle feuille de route pour les Conseils Généraux ?", Environnement et technique, n² 258, p. 26-28.

Baumgärtner S., de Swaan Arons J., 2003, "Necessity and Inefficiency in the Generation of Waste: A Thermodynamic Analysis", Journal of industrial ecology, vol. 7, n² 2. p.113-123.

Bertolini G., 2003, "Le coût social des consommations d'espace : Approche méthodologique et étude de cas relative au traitement des déchets", Déchets sciences et techniques, $n^{\circ}$ 30, p. 3-10.

Boux C., De Kerangal A.S., Courboulay C., Bertagna P.L., 2010, "La planification, une compétence régionale en Île-de-France", Techniques Sciences Méthodes, n 9, p. 24-31.

Casteigts M., 2009, "Optimisation du développement durable et management territorial stratégique : de la gouvernance locale à la transaction sociale", Vertigo - la revue électronique en sciences de l'environnement [En ligne], Hors série 6 | novembre 2009, mis en ligne le 09 novembre 2009, consulté le 27 novembre 2012. URL : http://vertigo.revues.org/8987 ; DOI : $10.4000 /$ vertigo.8987

Chang N.B., Schuler R.E., 1991, "Optimal pricing of the sanitary landfill use over time", Journal of Resource Management and Technology, vol. 19, nº 1, p. 14-24.

Conseil Général Essonne, 2002, Plan départemental d'élimination des déchets ménagers et assimilés de l'Essonne, Essonne, $115 \mathrm{p}$.

Conseil Général Seine-et-Marne, 2004, Révision du plan départemental d'élimination des déchets ménagers et assimilés de Seine-et-Marne, Seine-et-Marne, 151 p.

Conseil Général Seine-et-Marne, 2009, Délibération du Conseil Général n¹-14, Seine-et-Marne, 27 mars 2009.

Conseil Général Seine-Saint-Denis, 2005, Plan départemental de gestion et d'élimination des déchets ménagers et assimilés de la Seine-Saint-Denis, Seine-Saint-Denis, 59 p. 
Conseil Général Val-d'Oise, 2006, Plan départemental d'élimination des déchets ménagers et assimilés, Val-d'Oise, 68 p.

Conseil Général Val-d'Oise, 2009, Délibération du Conseil Général n²-12, Val-d'Oise, 24 mars 2009.

Conseil Régional d'île-de-France (CRIdF), 2007, Vers un plan régional d'élimination des déchets ménagers et assimilés: attentes et réflexions des acteurs, des associations et des élus régionaux, île-deFrance, $74 \mathrm{p}$.

Conseil Régional d'île-de-France (CRIdF), 2007, Vers un plan régional d'élimination des déchets ménagers et assimilés: Enjeux et perspectives, CRIdF - Unité aménagement durable - Direction de l'environnement, île-de-France, $94 \mathrm{p}$.

Conseil Régional d'île-de-France (CRIdF), 2008, Journée de concertation décentralisées - Synthèse et enseignements pour l'élaboration du PREDMA, île-de-France, $111 \mathrm{p}$.

Conseil Régional d'île-de-France (CRIdF), 2009, Plan régional d'élimination des déchets ménagers et assimilés d'île-de-France, Île-de-France, $190 \mathrm{p}$.

Fischhendler I., Feitelson E., 2003, "Spatial Adjustment as a Mechanism for Resolving River Basin Conflicts: U.S.-Mexico case", Political Geography, Vol. 25, n 5, p. 547-573.

Lomborg B., 2004, L'écologiste sceptique : le véritable état de la planète, Paris, Le Cherche Midi, 742 p. Lund J.R., 1990, "Pricing solid and hazardous waste landfill capacity", Journal of urban planning and development, Vol. 116, $\mathrm{n}^{\circ} 1$, p. 17-33.

Ministère de l'Ecologie, de l'Energie, du Développement Durable et de la Mer (MEEDDM), 2009, Plan d'action déchets 2009-2012, 14 p.

Méry J., Mtibaa R., Torre A., 2009, "Dynamique de proximité et gestion des déchets : application à la mise en décharge", 6èmes journées de la proximité, le temps des débats, Poitiers, 14-16 octobre 2009.

Mtibaa R., Méry J., Torre A., 2009, "Conflits autour des ISDND : quelles conséquences pour la gouvernance territoriale des déchets", XLVI ${ }^{\text {ime }}$ colloque de l'ASRDLF, Clermont-Ferrand, juillet 2009. Observatoire Régional des Déchets d'île-de-France (ORDIF), 2008, La planification régionale des déchets d'Ile-de-France, interview de Michel Vampouille, La lettre de l'ORDIF, $\mathrm{n}^{\circ} 4$.

Préfecture Hauts-de-Seine, 2000, Plan de gestion des déchets ménagers et assimilés, Hauts-de-Seine, $129 \mathrm{p}$.

Préfecture de Paris, 2001, Plan départemental d'élimination des déchets ménagers et assimilés de Paris, Paris, $44 \mathrm{p}$.

Préfecture Val-de-Marne, 2003, Plan départemental d'élimination des déchets ménagers et assimilés du Val-de-Marne, Val-de-Marne, $41 \mathrm{p}$.

Préfecture Yvelines, 2001, Plan départemental d'élimination des déchets ménagers et assimilés, Yvelines, $112 \mathrm{p}$.

Ready M.J., Ready R.C., 1995, "Optimal Pricing of Depletable, Replaceable Resources: The Case of Landfill Tipping Fees", Journal of Environmental Economics and Management, Vol. 28, n³, p. 307-323.

Rocher L., 2006, Gouverner les déchets - Gestion territoriale des déchets ménager et participation publique , Thèse de doctorat en Aménagement de l'espace - Urbanisme, Université François Rabelais, Tours, $444 \mathrm{p}$.

Schumacher E.F., 1973, Small is beautiful : a study of economics as if people mattered, Blond and Briggs, $288 \mathrm{p}$. 
Sénat, 2010, Rapport d'information fait au nom de la mission commune d'information sur le traitement des déchets, $\mathrm{n}^{\circ} 571,183 \mathrm{p}$.

Torre A., 2010, "Conflits environnementaux et territoires", in Zuindeau B. (éd.), Développement durable et territoire, Villeneuve d'Ascq, Presses Universitaires du Septentrion, p. 109-121.

\section{ANNEXES}

\section{Glossaire de certains termes utilisés}

ADEME : Agence de l'Environnement et de la Maîtrise de l'Energie

APPACE : Association pour le Plateau Agricole de Centre Essonne

CAPEFA : Confédération des Associations de Protection de l'Environnement de Fontainebleau et Alentours

CRIdF : Conseil régional d'Île-de-France

DIB : Déchets Industriels Banals. C'est l'ensemble des déchets non inertes et non dangereux générés par les entreprises, industriels, artisans et prestataires de services. Selon le Code de l'Environnement, l'entreprise reste responsable du devenir de ses déchets jusqu'à leur élimination.

DMA : Déchets Ménagers et Assimilés

FFOM : Fraction Fermentescible des Ordures Ménagères

FNADE : Fédération Nationale des Activités de la Dépollution et de l'Environnement

Groupe des élus CACR : Groupe des élus Communistes, Alternative Citoyenne et Républicain du Conseil Régional d'Île-de-France

ISDND : Installations de Stockage de Déchets Non Dangereux

NIMBY : Not In My Back Yard

OMR : Ordures Ménagères Résiduelles

PDEDMA : Plan Départemental d'Elimination des Déchets Ménagers et Assimilés

PREDMA : Plan Régional d'Elimination des Déchets Ménagers et Assimilés

REFIOM : Résidus d'Epuration des Fumées d'Incinération d'Ordures MénagèresUIOM :

Unité d'Incinération d'Ordures Ménagères

\section{NOTES}

1. Il s'agit des sites de Claye-Souilly (77) et du Plessis-Gassot (95), ouverts respectivement depuis 1972 et 1971. Leurs autorisations d'exploitation ont été prolongées respectivement jusqu'à 2026 et 2027. Chacun de ces sites est autorisé pour une capacité d'enfouissement de $1100 \mathrm{kt} / \mathrm{an}$.

2. Phénomène souvent décrit par l'acronyme NIMBY : Not In My Back-Yard. 
3. En 2010, 9 ISDND sont en exploitation (en plus des deux grandes), leurs capacités autorisées varient entre 80 et $220 \mathrm{kt} / \mathrm{an}$ (situation en 2010). La moyenne des capacités autorisées sur ces 9 installations est de $145 \mathrm{kt} / \mathrm{an}$.

4. A cette date, aussi bien l'autorisation d'exploitation de Claye-Souilly (77) que du Plessis-Gassot (95) auraient expirées.

5. Calcul fait sans tenir compte des 9 autres ISDND en exploitation pour une capacité d'enfouissement totale de $1505 \mathrm{kt} / \mathrm{an}$, également appelées à fermer et dont la question de la prolongation ou remplacement se pose.

6. Le PREDMA retient cette date comme celle qui marque le moment où les besoins seront supérieurs aux capacités d'enfouissement.

7. DMA: Déchets Ménagers et Assimilés, la gestion de ces déchets relève directement et exclusivement des collectivités territoriales. Comme le précisent les articles L. 2224-13 et 14 du code général des collectivités territoriales, les communes ou leurs groupements doivent assurer l'élimination des déchets des ménages, mais aussi des déchets qu'elles produisent elles-mêmes (espaces verts, voirie) et des déchets d'origine commerciale ou artisanale ayant les mêmes caractéristiques que les déchets ménagers.

8. Cette loi est la retranscription de la directive $n^{\circ} 75-442$ du 15 juillet 1975 de la Communauté Economique Européenne.

9. "Les "affaires" dénoncées portent généralement sur des catégories de déchets particuliers - en l'occurrence les déchets industriels - et des pratiques considérées comme immorales telles les transferts transfrontaliers. Mais progressivement la qualification de la situation "de crise" va s'étendre aux déchets ménagers jusqu'alors relativement épargnés en raison de leur apparente banalité" (Rocher, 2006).

10. "C'est au cours de la décennie 1980 que les conflits déclenchés lors de projets d'implantation d'équipements -quelles que soit la nature des déchets- se multiplient et deviennent systématique. Jusqu'alors épisodiques et considérés comme un problème à la marge, ces contestations deviennent au tournant 1990 une donnée centrale de la politique déchets dans la mesure où elles rendent difficile voire impossible la réalisation d'exutoires et engendrent une situation de pénurie des capacités" (Rocher, 2006).

11. Ce principe figure de manière implicite dans l'article L541-1 du code de l'environnement, où un des objets de la gestion des déchets doit être "d'organiser le transport des déchets et de le limiter en distance et volume".

12. Il est intéressant de remarquer que le Conseil Général de l'Essonne a pris en charge l'élaboration du PDEDMA alors qu'il n'en avait pas encore l'obligation.

13. Directive 94/62/CE du 20 décembre 1994.

14. Décret 96-1008 du 18 novembre 1996, circulaire ministérielle du 28 avril 1998.

15. Loi n 2004-809 du 13 août 2004.

16. Loi n92-646 du 13 juillet 1992.

17. La définition déduite est celle obtenue à partir des choix de gestion des déchets indiqués dans le plan.

18. SYCTOM : Avec la décentralisation, en 1984, 60 communes se sont regroupées dans un syndicat intercommunal pour traiter ensemble les déchets de leurs habitants. De cette union est né le plus important syndicat européen de traitement et de valorisation des déchets : le SYCTOM de l'agglomération parisienne, Syndicat intercommunal de traitement des ordures ménagères de l'agglomération parisienne. En 2011, Le SYCTOM devient le Syctom, l'agence métropolitaine des déchets ménagers.

19. Val-de-Marne, Hauts-de-Seine et Seine-Saint-Denis.

20. Selon le bassin versant de la commune, les déchets sont orientés vers les UIOM d'Ivry-surSeine(94), d'Issy-les-Moulineaux (92), de Saint-Ouen (93), ou vers le centre de transfert de 
Romainville (93) qui les achemine en partie vers ses usines et en partie vers les ISDND de ClayeSouilly (77) et du Plessis-Gassot (95).

21. Importation de déchets ménagers en provenance du SYCTOM. Exportation de la totalité des REFIOM, d'une partie des mâchefers et de la totalité des déchets ultimes à stocker en CET de classe II.

22. $100 \mathrm{kt} / \mathrm{an}$ dont $26 \mathrm{kt} / \mathrm{an}$ d'encombrants et $74 \mathrm{kt} / \mathrm{an}$ de DIB. La quantité de DIB est estimée selon l'hypothèse que plus de $40 \%$ des $250 \mathrm{kt} / \mathrm{an}$ de DIB produits dans le département et mis en décharge en dehors de ses frontières à ce moment-là présentaient un caractère ultime.

23. Par la loi de décentralisation "démocratie de proximité" du 27 février, deux compétences ont notamment été transférées aux Régions en matière de gestion de déchets, le Plan régional d'élimination des déchets spéciaux (PREDIS), rebaptisé Plan régional d'élimination des déchets dangereux (PREDD) par le Conseil Régional au vu de son élargissement à l'ensemble des déchets dangereux et le Plan régional d'élimination des déchets d'activités de soins (PREDAS).

24. Echéance fixée par la loi Royal sur l'arrêt de l'acheminement des déchets bruts en décharges.

25. Les impacts environnementaux, la prévention des déchets "du concepteur à l'utilisateur», l'optimisation du traitement et les nouvelles technologies, le transport des déchets, les filières de recyclage et de réemploi, la communication autour de la gestion des déchets, la tarification et le coût global de la gestion des déchets.

26. Plénière du PREDMA le 12 octobre 2007. Les journées de concertation décentralisées du PREDMA en 2008.

27. En matière de déchets, la plupart des pays industrialisés possèdent une législation fondée sur le principe des 3R: Réduire, Réutiliser, Recycler.

28. Code de l'environnement - Livre V - Titre IV - Chap Ier - Section 3 - Article 541.14.

29. Pour les incinérateurs l'enjeu est la recherche d'une proximité avec le chauffage urbain, pour les ISDND c'est la valorisation du biogaz.

30. Par exemple, le PDEDMA de l'Essonne déclarait laisser toute initiative de création ou l'extension de sites de traitement et d'élimination de DIB à l'appréciation des opérateurs privés (Conseil Général Essonne, 2002). Il est à noter que l'Essonne figure parmi les rares cas où la compétence en charge du plan s'est penchée sur la question des DIB, question rarement abordée. 31. Les besoins de stockage ont été évalués avec comme horizon temporel 2024, le plan ne porte que sur une période de 10 ans autrement dit 2019.

\section{RÉSUMÉS}

La gestion des déchets franciliens est un cas d'étude intéressant dans l'analyse des causes et effets des changements d'échelle de gouvernance territoriale, les huit plans de gestion départementaux ayant été refondus en un seul plan de gestion régional. Les raisons de ce changement d'échelle, et les conséquences dans le cas particulièrement sensible du point de vue de la gouvernance territoriale de la mise en décharge des déchets non dangereux, sont présentées et analysées dans cet article. On en déduit que le changement d'échelle ne modifie pas fondamentalement la problématique du stockage des déchets en île-de-France, mais permet de leur donner un cadre institutionnel adéquat.

The management of the waste of the Ile-de-France region is an interesting case study for analysing causes and effects of a scale change on territorial governance: eight local plans have 
been recently renewed for a unique regional plan. The reasons of this change and the consequences on the landfilling of non-dangerous waste (a typically controversial application of territorial governance) are presented and analyzed in the paper. The change of scale does not modify the fundamentals of regional waste landfilling, but allows for an adequate institutional frame.

\section{INDEX}

Mots-clés : déchets, Conseil Régional d'île-de-France, gouvernance, installations de stockage de déchets non dangereux, plans départementaux d'élimination des déchets ménagers et assimilés Keywords : wastes, governance, île-de-France Régional Council, scale, wastes planning, municipal solid wastes landfill

\section{AUTEURS}

\section{RYM MTIBAA}

Rym Mtibaa, économiste de l'environnement, a travaillé pendant quatre ans (2007-2010) sur les aspects socioéconomiques liés aux installations de stockage de déchets non dangereux au sein d'Irstea, où elle a fournit le travail faisant l'objet de cet article. Elle poursuit actuellement sa carrière en tant que chargée de mission prévention et gestion des déchets au sein du Conseil Régional d'Ile-de-France. rym.mtibaa@gmail.com

\section{JACQUES MERY}

Jacques Méry est ingénieur de recherche en socio-économie de l'environnement appliquée à la gestion des déchets non dangereux et travaille sur l'évaluation et la gestion des impacts environnementaux et sociaux du stockage. Irstea, UR HBAN, CS 10030, 92761 Antony, jacques.mery@irstea.fr

\section{ANDRÉ TORRE}

André Torre est directeur de recherche en économie, responsable de l'équipe Proximités de I'UMR INRA-AgroParisTech SADAPT, et travaille sur l'économie des territoires et les conflits d'usage et de voisinage. UMR INRA-AgroParisTech SADAPT, 16 rue Claude Bernard, 75231 Paris, torre@agroparistech.fr 